\title{
UNCERTAINTY MODELLING AND LIMIT STATE RELIABILITY OF TUNNEL SUPPORTS UNDER SEISMIC EFFECTS
}

\author{
Mallika $S^{1}$, Srividya. $A^{2}$, Venkatachalam. $G^{3}$ \\ ${ }^{I}$ Research Scholar, Reliability Engineering Group, IIT Bombay, Powai, Mumbai, India, mallika@iitb.ac.in \\ ${ }^{2}$ Guest Professor, University College, Haugesund, Norway, asvidya88@gmail.com \\ ${ }^{3}$ Emeritus Fellow, Department of Civil Engineering, IIT Bombay, Powai, Mumbai, India, gvee@iitb.ac.in
}

\begin{abstract}
Underground openings and excavations are increasingly being used for civilian and strategic purposes all over the world. Recent earthquakes and resulting damage have brought into focus and raised the awareness for aseismic design and construction. In addition, underground tunnels, particularly, have distinct seismic behaviour due to their complete enclosure in soil or rock and their significant length. Therefore, seismic response of tunnel support systems warrant closer attention. The geological settings in which they are placed are often difficult to describe due to limited site investigation data and vast spatial variability. Therefore, the parameters which govern the design are many and their variabilities cannot be ignored. A solution to this issue is reliability based analysis and design. These real conditions of variability can only be addressed through a reliability based design. The problem addressed here is one of reliability-based analysis of the support system of an underground tunnel in soil. Issues like the description of the interaction between the tunnel lining and the surrounding medium, the type of limit state that would be appropriate, the nonavailability of a closed form performance function and the advantages of response surface method [RSM] are looked into. Both static and seismic environment with random variability in the material properties are studied here. Support seismic response is studied in terms of thrust, moment and shear forces in the lining. Interactive analysis using finite element method [FEM], combined with RSM and Hasofer-Lind reliability concept to assess the performance of the tunnel support, has proven useful under real field situations.
\end{abstract}

Index Terms: Tunnel, Reliability, Random, Seismic

\section{INTRODUCTION}

The need today in civil engineering, more than ever before, is toward providing economical designs commensurate with safety. This requires a careful consideration of variabilities in loads, geometry and material properties governing the behavior of the given structure. Reliability analysis is best suited for this. While considerable developments have taken place in application of reliability concepts in structural engineering, there is a need for more studies in geotechnical applications.

The importance of reliability analysis is best illustrated in the evaluation of slope stability. Several researchers have contributed to the understanding of slope reliability $[1,2,3,4]$. The measure normally adopted for expressing safety is the factor of safety, which is evaluated from the relative magnitudes of resistance (capacity) and load (demand) as FS = Capaity (C) / Demand (D). Since capacity and demand cannot be evaluated with certainty, assuming a random variation, a ratio of expected values of capacity and demand called Central factor of safety is preferred. However, when material properties exhibit substantial variabilities, the minimum factor of safety may not be the correct measure. A better measure of safety, therefore, is the Reliability Index, $\beta$, which accounts for the randomness of FS itself. It is defined as the number of standard deviations by which the expected value of FS exceeds a critical or acceptable value. This may be expressed as (Eq.1):

$\beta=\frac{E(F S)-1}{\sigma(F S)}$

Assuming normal distribution of capacity and demand, another parameter, Probability of failure, is expressed as (Eq.2):

$p_{f}=\frac{1}{2}-\varphi(\beta)=\varphi(-\beta)$

Where, $\varphi(\beta)$ is the standard normal probability.

However, the variable values corresponding to the least value of $\beta$ would be the most critical [5]. The Hasofer-Lind reliability index $\beta_{\mathrm{HL}}$ is defined as the shortest distance from the origin of the reduced variable space to the limit state function $g=0$.

Consider a limit state function $\mathrm{g}\left(\mathrm{X}_{1}, \mathrm{X}_{2} \ldots \ldots \mathrm{X}_{\mathrm{n}}\right)$ with uncorrelated $X_{i}$ variables. The Hasofer-Lind reliability index is determined by the following steps:

1. The reduced variables $\left\{Z_{1}, Z_{2}, \ldots, Z_{n}\right\}$ are first determined as (Eq.3): 
$Z_{i}=\left(X_{i}-\mu_{x i}\right) / \sigma_{x i}$

2. The limit state function is then redefined in terms of these reduced variables.

3. The reliability index is the shortest distance from the origin in the n-dimensional space of reduced variables to the curve corresponding to the limit state function described by $\mathrm{g}(\mathrm{Z}$, $\left.\mathrm{Z}_{2}, \ldots, \mathrm{Z}_{\mathrm{n}}\right)=0$.

The Hasofer-Lind reliability index $\beta_{\mathrm{HL}}$ can be expressed in matrix form as (Eq. 4):

$$
\beta_{H L}=\min \sqrt{\left((X-\mu)^{T} C^{-1}(X-\mu)\right)}
$$

where $\mathrm{x}$ vector represents the $\mathrm{n}$ random variables; $\mu$ is the vector of their mean values; and $\mathrm{C}$ is the corresponding covariance matrix.

The minimization of $\mathrm{Eq}$ (4) is performed subject to the constraint $\mathrm{g}(\mathrm{x}) \leq 0$ where the limit state surface $\mathrm{g}(\mathrm{x})=0$ separates the n-dimensional domain of random variables into two regions: a failure region $\mathrm{F}$ represented by $\mathrm{g}(\mathrm{x}) \leq 0$ and $\mathrm{a}$ safe region given by $g(x)>0$. A schematic of the Hasofer-Lind reliability estimation is given in Fig.1, where superscript $\mathrm{N}$ represents normative values and $\theta$ represents an angle as shown.

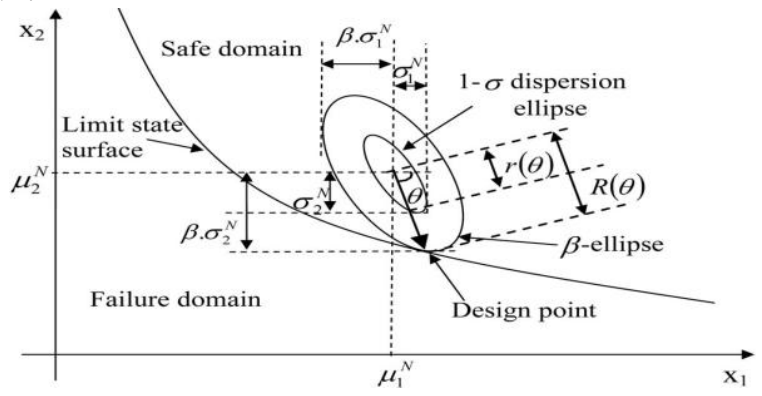

Fig-1: Schematic of Hasofer-Lind Reliability Index estimation

Reliability analysis has also been done for Piles subjected to axial and lateral loads [6]. Empirical, analytical and numerical methods are available for analysis and design of tunnels. The empirical methods such as the geomechanical methods consider rock mass characteristics, which are otherwise very difficult to model. Authors of [7] have brought out the significance of seismic analysis, though the analytical methods, such as those employed by them, generally consider ideal simplified situations. Numerical methods have come to be employed widely during the past few decades due to their ability to model complex geometry, loading, stress-strain relationships and construction sequences [8, 9]. Apart from the rigourousness of the method of analysis, consideration of material properties, especially their variabilties, also merits close attention and the present study is an attempt in that direction.

\section{RESPONSE SURFACE METHODOLOGY}

Following are some uncertainties surrounding the seismic analysis of the underground openings.

- Uncertainties in characterizing the seismic event

- Uncertainties in structural and material modeling

- Uncertainties/errors in method of analysis and performance criteria

In the current study, the response of the underground opening under static and seismic loading is analyzed considering the ultimate limit state (ULS) as the performance criterion and material properties as random variables (see for example, Eq. 5). The response surface methodology (RSM) is adopted herein in order to study the response of the tunnel lining considering the effect of the randomness of the ground material parameters. Based on the observed data from the system, an empirical model, which is a polynomial function of the random variables, is built using regression analysis of the selected deterministic analysis results. This polynomial, called the response surface, can serve as a basis for further simulations and hence, for a better estimation of the probability of failure (Eq. 6). The true response, $\mathrm{y}$, of a system is given by [10]:

$y=f\left(\xi_{1}, \xi_{2}, \ldots, \xi_{k}\right)+\varepsilon$

Where $\xi_{i}$ are the independent variables and $\varepsilon$ is the modeling error. The second-order polynomial approximation of the true response function involving two factors is:

$y=\beta_{0}+\beta_{1} x_{1}+\beta_{2} x_{2}+\beta_{12} x_{1} x_{2}+\beta_{11} x_{1}^{2}+\beta_{22} x_{2}^{2}$

Where $x_{i}$ are called coded variables, which are transformed values of the actual variables $\xi_{\mathrm{i}}$, to the domain of $[-1,1]$ and $\beta_{\mathrm{ij}}$ are called regression coefficients. Fig. 2 shows the number of analyses required and the values of the variables to be used in the case of three factors ( $a, b$ and $c)$.

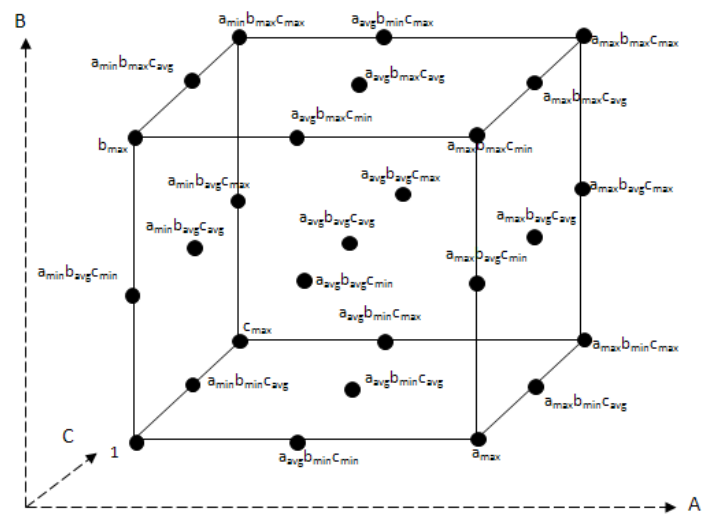

Fig-2: Three level three factor experiment 


\section{STATEMENT OF PROBLEM}

In order to evaluate the effect of uncertainties, a real tunnel and the earthquake to which it was subjected or considered. The case study chosen concerns a tunnel of $6 \mathrm{~m}$ diameter and overburden depth between 21.0 and $25.3 \mathrm{~m}$ [11].

The center of the studied section of the tunnel is approximately $24 \mathrm{~m}$ deep with a $21 \mathrm{~m}$ thick overburden and is embedded in the Shongsan formation which comprises six alternating silty sand and silty clay layers. A typical cross section of the formations, the alignment of the tunnel and related information are given in (Fig. 3). The material properties of the formation and that of the tunnel lining are listed in Table 1 and Table 2 respectively.

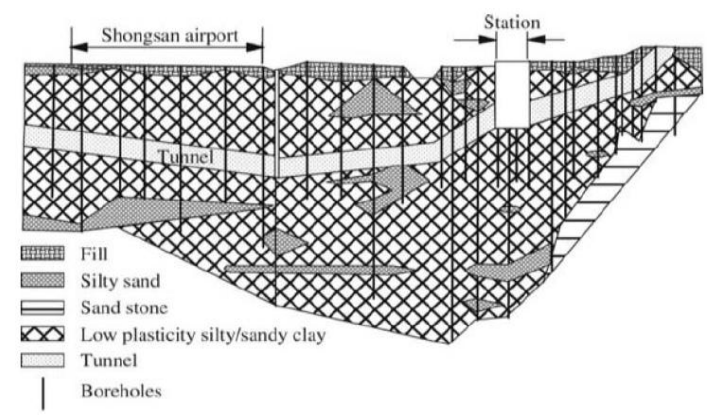

Fig-3: Cross-section of the soil stratification at Shongsan airport tunnel site (Gui and Chien, 2006)

Table-1: Material properties of the ground medium.I

\begin{tabular}{|c|c|}
\hline Ground properties & Mean values \\
\hline Bulk unit weight $\gamma\left(\mathrm{kN} / \mathrm{m}^{3}\right)$ & 18 \\
\hline Modulus of elasticity $\mathrm{E}_{\mathrm{stat}}(\mathrm{MPa})$ & 28 \\
\hline Poisson's ratio $v$ & 0.3 \\
\hline Cohesion c $\left(\mathrm{kN} / \mathrm{m}^{2}\right)$ & 30 \\
\hline Friction angle $\varphi($ degrees $)$ & 31 \\
\hline Modulus of elasticity $\mathrm{E}_{\mathrm{dyn}}(\mathrm{MPa})$ & 253 \\
\hline
\end{tabular}

Table-2: Tunnel lining parameters (a) a Steel (b) Concrete

\begin{tabular}{|c|c|}
\hline \multicolumn{2}{|c|}{ Grade 60 Steel (16 Nos.) } \\
\hline Modulus of elasticity of steel (MPa) & 210000 \\
\hline Poisson's ratio & 0.2 \\
\hline Tensile strength (MPa) & 420 \\
\hline Shotcrete Thickness (m) & 0.3 \\
\hline
\end{tabular}

(a)

\begin{tabular}{|c|c|}
\hline \multicolumn{2}{|c|}{ Shotcrete Lining } \\
\hline Modulus of shotcrete (MPa) & 30500 \\
\hline Poisson's ratio & 0.2 \\
\hline Tensile strength (MPa) & 0.36 \\
\hline Compressive strength (MPa) & 42 \\
\hline
\end{tabular}

(b)

\section{METHODOLOGY}

Interestingly, when material property variation is the main consideration, reliability analysis requires only a set of deterministic analyses using different combinations of material properties. Hence, the methodology used consists essentially of three steps. In the first step, a series of deterministic analyses is carried out. Since there are three variables (factors), considering mean and \pm standard deviation, the number of combinations works out to $(3)^{3}=27$. Hence, 27 deterministic analyses are carried out using PLAXIS and thrust, moment and shear are calculated in the lining. Next, using these values and RSM, an equation is built for each of these quantities in terms of three variables. This serves as an equivalent alternative function for the unknown performance function. In the third step, the resulting equations are used to arrive at the least reliability index.

Two cases as shown in Table 3 have been considered. The objective of choosing the two cases is to identify the effect of the random nature of the material parameters (cohesion c, Elastic modulus, $\mathrm{E}$ and angle of internal friction $\varphi$ ) on the lining performance, under ultimate and serviceability conditions within static as well as seismic environment. To perform the reliability analysis the material properties of the ground are considered as normally distributed random variables with the mean and Coefficient of Variation (COV) as shown in Table 4. For the chosen COV, there was no likelihood of the normal variables turning negative. However, for a higher $\mathrm{COV}$, say more than $30 \%$, there is a possibility of normal variables turning negative. In such cases, assumption of a lognormal distribution would help. The effect of their randomness on the performance of the tunnel support system is then studied using RSM, where, the response surface built is evaluated to obtain the least reliability index $\beta_{\mathrm{HL}}$ through repeated iterations and subsequent convergence.

Table-3: Cases studied

\begin{tabular}{|c|c|c|}
\hline Case & ULS & SLS \\
\hline 1. Static & $\begin{array}{c}\text { Thrust, Moment, Shear } \\
\text { force }\end{array}$ & Deformation \\
\hline 2. Seismic & $\begin{array}{c}\text { Thrust, Moment, Shear } \\
\text { force }\end{array}$ & - \\
\hline
\end{tabular}

Table-4: Properties of chosen Normal Random variables.

\begin{tabular}{|c|c|c|}
\hline Ground properties & $\begin{array}{c}\text { Mean } \\
\text { values }\end{array}$ & $\begin{array}{c}\text { COV } \\
(\%)\end{array}$ \\
\hline Modulus of elasticity $\mathrm{E}_{\text {stat }}(\mathrm{MPa})$ & 28 & 13 \\
\hline Modulus of elasticity $\mathrm{E}_{\mathrm{dyn}}(\mathrm{MPa})$ & 253 & 13 \\
\hline Cohesion c $\left(\mathrm{kN} / \mathrm{m}^{2}\right)$ & 30 & 30 \\
\hline Friction angle $\varphi($ degrees $)$ & 31 & 10 \\
\hline
\end{tabular}




\subsection{Performance Functions using RSM}

Based on the limit states defined for the two cases listed above, the performance functions for the thrust, moment, shear force and deformation responses are as follows:

$$
\begin{aligned}
& -U L S \text { of thrust: } \mathrm{Nc} / \mathrm{N}-1=0 \\
& \text {-ULS of moment: } \mathrm{Mc} / \mathrm{M}-1=0 \\
& \text {-ULS of shear : Qc/Q-1=0 } \\
& \text {-SLS of deformation: } \mathrm{yc}-\mathrm{y}=0
\end{aligned}
$$

Here, the notations $\mathrm{Nc}, \mathrm{Qc}$, Mc correspond to the ultimate capacity of thrust, moment and shear force calculated based on ACI 318-05 [12] for the given lining material properties as listed in Table 5. The serviceability limit state is defined by limiting the displacement yc of the lining to $5 \mathrm{~mm}$.

Table-5: Structural capacity of the tunnel lining

\begin{tabular}{|c|c|}
\hline Thrust capacity Nc $(\mathrm{kN} / \mathrm{m})$ & 7993 \\
\hline Moment capacity $\mathrm{Mc}(\mathrm{kNm} / \mathrm{m})$ & 283 \\
\hline Shear capacity Qc $(\mathrm{kN} / \mathrm{m})$ & 3888 \\
\hline
\end{tabular}

For the seismic analysis, the accelerogram of the earthquake that occurred in November 14, 1986 with a magnitude of 7.8 at a distance of approximately $120 \mathrm{~km}$ from the airport and at a depth approximately $34 \mathrm{~m}$ is used. The peak ground acceleration PGA is 0.13g (Fig 4). Fig. 5 shows the FE model used for the dynamic analysis. In order to apply RSM, 27 sample points were taken initially as shown in Fig.1. The numbers of experiments were chosen [13] such that the convergence of the order $10^{-1}$ is attained within three to four iterations. The selected sample points are $\left(c_{i}, \varphi_{i}, E_{i}\right), \quad\left(c_{i}+1.2 \sigma_{c}, \varphi_{i}, E_{i}\right), \quad\left(c_{i}-\right.$ $\left.0.3 \sigma_{\mathrm{c}}, \varphi_{\mathrm{i}}, \mathrm{E}_{\mathrm{i}}\right),\left(\mathrm{c}_{\mathrm{i}}, \varphi_{\mathrm{i}}+1.4 \sigma_{\varphi}, \mathrm{E}_{\mathrm{i}}\right),\left(\mathrm{c}_{\mathrm{i}}, \varphi_{\mathrm{i}}-0.3 \sigma_{\varphi}, \mathrm{E}_{\mathrm{i}}\right),\left(\mathrm{c}_{\mathrm{i}}, \varphi_{\mathrm{i}}, \mathrm{E}_{\mathrm{i}}+\sigma_{\mathrm{E}}\right)$ and $\left(c_{i}, \varphi_{i}, E_{i}-\sigma_{E}\right)$

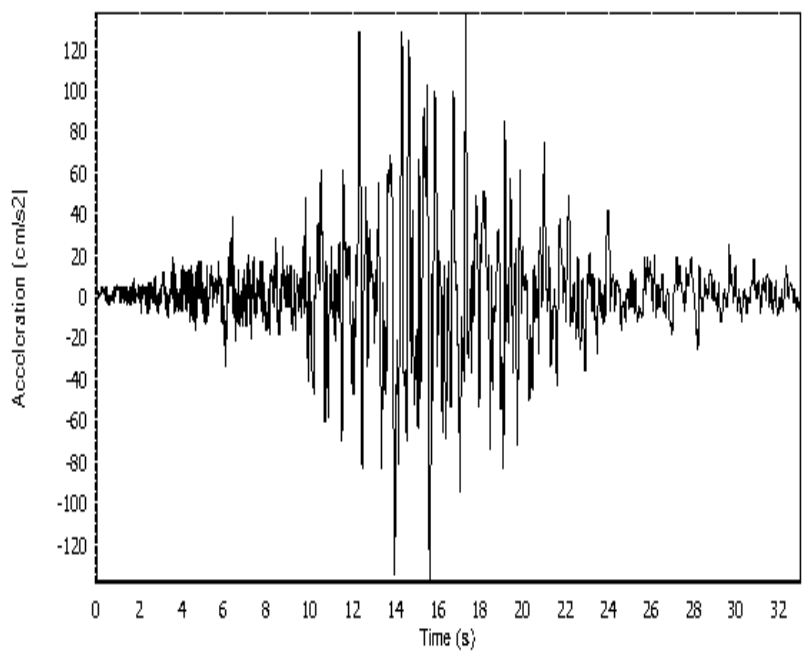

Fig-4: Accelerogram of Taiwan Earthquake, November14, 1986 [14] (National Geophysical Data Centre)

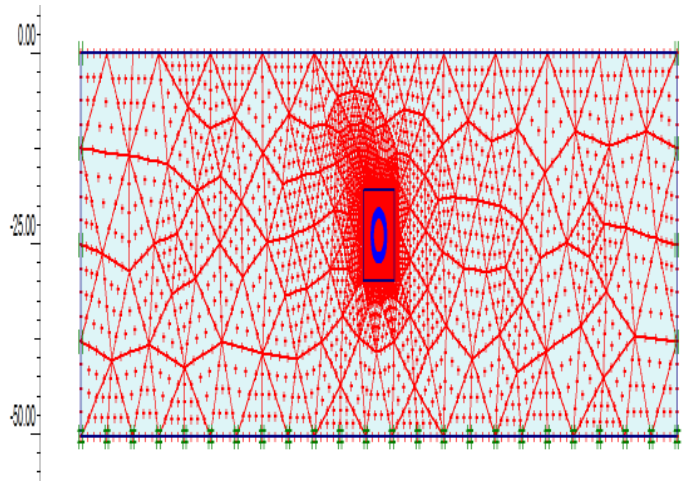

Fig-5: Finite Element model used for seismic analysis

\section{RESULTS AND OBSERVATIONS}

The results are analyzed and discussed in terms of thrusts, moments, shear and displacements. The associated reliabilities under appropriate limit states and corresponding critical material property combinations are examined.

Table 6 illustrates the iterations conducted for the RSM used in obtaining the reliability index for the seismic case. Table 7 illustrates the minimum reliability obtained for the displacement response.

Table-6: Iterations performed to obtain minimum reliability for (a) thrust, (b) moment and (c) shear response

\begin{tabular}{|c|c|c|c|c|}
\hline \multirow{2}{*}{} & \multirow{2}{*}{$\begin{array}{c}\text { Initial } \\
\text { values }\end{array}$} & \multicolumn{3}{|c|}{$\begin{array}{c}\text { Iteration \# for thrust } \\
\text { response }\end{array}$} \\
\cline { 3 - 5 } & & $1^{\text {st }}$ & $2^{\text {nd }}$ & $\mathbf{3}^{\text {rd }}$ \\
\hline$\beta$ & 0 & 1.08 & 3.60 & $\mathbf{1 . 7 0}$ \\
\hline $\mathrm{c}(\mathrm{kPa})$ & 30 & 23.63 & 49.59 & $\mathbf{4 8 . 8 5}$ \\
\hline$\Phi(\mathrm{deg})$ & 31 & 33.60 & 32.95 & $\mathbf{3 5 . 2 0}$ \\
\hline $\mathrm{E}(\mathrm{MPa})$ & 253 & 441.75 & 462.46 & $\mathbf{3 6 8 . 6 0}$ \\
\hline
\end{tabular}

\begin{tabular}{|c|c|c|c|c|}
\hline & \multirow{2}{*}{$\begin{array}{l}\text { Initial } \\
\text { values }\end{array}$} & \multicolumn{3}{|c|}{ Iteration \# for shear response } \\
\hline & & $1^{\text {st }}$ & $2^{\text {nd }}$ & $3^{\text {rd }}$ \\
\hline$\beta$ & 0 & 0.003 & 0.56 & 0.31 \\
\hline $\mathrm{c}(\mathrm{kPa})$ & 30 & 30.00 & 33.67 & 33.54 \\
\hline$\Phi(\operatorname{deg})$ & 31 & 31.00 & 30.04 & 30.35 \\
\hline $\mathrm{E}(\mathrm{MPa})$ & 253 & 334.32 & 344.18 & 331.22 \\
\hline
\end{tabular}

(a)

\begin{tabular}{|c|c|c|c|c|c|}
\hline & \multirow{2}{*}{$\begin{array}{c}\text { Initial } \\
\text { values }\end{array}$} & \multicolumn{4}{|c|}{ Iteration \# for moment response } \\
\cline { 3 - 6 } & 0 & $1^{\text {st }}$ & $2^{\text {nd }}$ & $3^{\text {rd }}$ & $\mathbf{4}^{\text {th }}$ \\
\hline$\beta$ & 0.82 & 3.47 & 2.84 & $\mathbf{2 . 0 7}$ \\
\hline $\mathrm{c}(\mathrm{kPa})$ & 30 & 25.20 & 13.17 & 2.75 & $\mathbf{1 . 4 2}$ \\
\hline$\Phi(\mathrm{deg})$ & 31 & 32.95 & 23.43 & 25.89 & $\mathbf{2 4 . 5 3}$ \\
\hline $\begin{array}{c}\mathrm{E} \\
(\mathrm{MPa})\end{array}$ & 253 & 425.07 & 485.84 & 493.88 & $\mathbf{5 6 9 . 2 0}$ \\
\hline
\end{tabular}

(b)

(c) 
Table-7: Iterations performed to obtain minimum reliability for displacement Response

\begin{tabular}{|c|c|c|c|c|}
\hline Reliability $\square \beta$ & 0 & 0.26 & 4.24 & $\mathbf{0 . 6 0}$ \\
\hline c $(\mathrm{kPa})$ & 30 & 28.74 & 20.64 & $\mathbf{2 4 . 2 8}$ \\
\hline$\Phi(\mathrm{deg})$ & 31 & 31.40 & 31.07 & $\mathbf{3 0 . 7 8}$ \\
\hline E $(\mathrm{MPa})$ & 28 & 27.35 & 12.64 & $\mathbf{1 2 . 5 5}$ \\
\hline
\end{tabular}

The minimum reliability corresponds to the last iteration for each response. The corresponding critical values are also listed alongside. It is also worth mentioning that the successive iterations do not result in perfect convergence for the given degree of tolerance. This could be attributed to the sampling points chosen and could be refined further to obtain proper convergence. The following are the equations ( Eq. 7 to 10) derived for the Thrust $(\mathrm{N})$, Moment $(\mathrm{M})$, Shear Force $(\mathrm{Q})$ and deformation of the tunnel lining:

$$
\begin{aligned}
N= & \mathrm{Nc} /(-6.360+0.496 \mathrm{c}+1.369 \phi-0.137 \mathrm{E}-0.008 \mathrm{c} \phi-0.0002 \mathrm{cE} \\
& \left.+0.0009 \phi \mathrm{E}-0.0014 \mathrm{c}^{2}-0.0102 \phi^{2}+0.0001 \mathrm{E}^{2}\right) \\
M= & \mathrm{Mc} /(203+143 \mathrm{c}-23.3 \phi+0.171 \mathrm{E}-21 \mathrm{c} \phi+0.826 \mathrm{cE} \\
& \left.-0.0838 \phi \mathrm{E}+2.26 \varphi^{2}-0.0002 \mathrm{E}^{2}\right) \\
Q= & \mathrm{Q}_{\mathrm{c}} / 14400-221 \mathrm{c}-561 \phi+0.737 \mathrm{E}-6.43 c \phi+0.206 c E \\
& -1.14 \phi E+5.19 c^{2}+19.1 \phi^{2}+0.0406 E^{2} \\
y= & \mathrm{yc}+282-16.4 \mathrm{c}+1.36 \phi-2.29 \mathrm{E}+0.0986 \mathrm{c} \phi-0.377 \mathrm{cE}+0.196 \phi \mathrm{E} \\
& +0.00279 \mathrm{c}^{2}-0.00575 \phi^{2}+0.463 \mathrm{E}^{2}
\end{aligned}
$$

A comparison of the static and seismic reliability levels of the lining response is given in Table 9.

Table-9: Comparison of reliability of the static and seismic case for the lining response

\begin{tabular}{|c|c|c|c|c|}
\hline \multirow{2}{*}{} & \multicolumn{4}{|c|}{ Thrust } \\
\cline { 2 - 5 } & $\beta$ & $\begin{array}{c}\mathrm{E} \\
(\mathrm{MPa})\end{array}$ & $\begin{array}{c}\mathrm{c}_{\mathrm{c}} \\
(\mathrm{kPa})\end{array}$ & $\varphi_{\mathrm{c}}(\mathrm{deg})$ \\
\hline Static & 1.77 & 2.02 & 34.60 & 23.40 \\
\hline Seismic & 1.70 & 368.56 & 48.80 & 35.20 \\
\hline
\end{tabular}

(a)

\begin{tabular}{|c|c|c|c|c|}
\hline \multirow{2}{*}{} & \multicolumn{4}{|c|}{ Moment } \\
\cline { 2 - 5 } & $\beta$ & $\begin{array}{c}\mathrm{E} \\
(\mathrm{MPa})\end{array}$ & $\begin{array}{c}\mathrm{c}_{\mathrm{c}} \\
(\mathrm{kPa})\end{array}$ & $\varphi_{\mathrm{c}}(\mathrm{deg})$ \\
\hline Static & 5.77 & 58.60 & 20.02 & 37.10 \\
\hline Seismic & 2.07 & 569.20 & 1.42 & 24.53 \\
\hline
\end{tabular}

(b)

\begin{tabular}{|c|c|c|c|c|}
\hline & \multicolumn{4}{|c|}{ Shear } \\
\cline { 2 - 5 } & $\beta$ & $\begin{array}{c}\mathrm{E} \\
(\mathrm{MPa})\end{array}$ & $\begin{array}{c}\mathrm{c}_{\mathrm{c}} \\
(\mathrm{kPa})\end{array}$ & $\varphi_{\mathrm{c}}(\mathrm{deg})$ \\
\hline Static & 1.30 & 25.51 & 34.93 & 34.74 \\
\hline Seismic & 0.31 & 331.22 & 33.54 & 30.35 \\
\hline
\end{tabular}

(c)

\subsection{Ultimate Limit State (ULS)}

The reliability of the lining system is analysed considering the thrust, moment and shear capacity of the lining under Ultimate limit state condition.

From the RSM based reliability analysis results (Tables 6, 7), the minimum reliability is obtained for the shear response where $\beta=0.31$ indicating a probability of failure $\left(p_{f}\right)$ of $37.83 \%$ under seismic condition. However for the thrust and moment response, where $\beta>1$, there is less probability of failure $\left(\mathrm{p}_{\mathrm{f}}<4 \%\right)$. This indicates that the chosen lining system is reliable against the randomness in ground material properties for the thrust and moment response. Table 9 also confirms that shear response under seismic case is poorer compared to the static case. This implies that strengthening against shear failure would be advisable.

\subsection{Serviceability Limit State (SLS)}

The displacement response indicates $\beta$ of 0.60 indicating a probability of failure under serviceability condition as $27.42 \%$.

\section{CONCLUSIONS}

The response surface methodology incorporating the randomness in the material properties of the ground and the subsequent reliability based analysis of the underground soilsupport interaction has been found to be useful. The study shows:

1. For the chosen lining system, the reliability decreases under seismic conditions considering the random nature of the ground properties chosen.

2. The reliability of the lining is low for the shear response under seismic case.

3. However considering the total response of the lining including the thrust, moment and shear for the ULS, the reliability is more than the SLS. Deformation and, hence, SLS is found to be the governing criterion of estimating the reliability of the support system as it provides the least reliability index.

4. Thus, reliability based analysis gives an optimum solution to the design of underground support system.

\section{ACKNOWLEDGEMENTS}

The computational and other support provided by IIT Bombay is gratefully acknowledged. 


\section{REFERENCES}

[1] Dodagoudar, G. R. and Venkatachalam, G. (2000). "Reliability analysis of slopes using fuzzy sets theory", Computers and Geotechnics, Vol. 27, pp. 101-115.

[2] Venkatachalam, G. (2004). "Hazard and risk evaluation of natural slopes", Key Note Paper presented at ASCE International Conf. Earth \& Space, 2004, Houston, Texas, USA.

[3] Venkatachalam, G. (2005). "Reliability and risk analysis of slopes and application to landslides", $28^{\text {th }}$ Annual Lecture of the Indian Geotechnical Society, delivered during IGC-2005, Ahmedabad.

[4] Sreedharan, M., Mathada, V.S. and Venkatachalam G.(2006). "Reliability analysis of rock slopes - case studies", Proc. Tenth International Conf. on Structural Engneeringand Construction (EASEC -10), Bangkok, Thailand, August 3-5, 2006, Vol.8, pp. 71-76.

[5] Hasofer, A.M. and Lind, N.C. (1974). "Exact and invariant second-moment code format", Journal of Engineering Mechanics, ASCE, Vol. 100(1), pp. 111121.

[6] Haldar, S and Babu, G.L.Sivakumar (2008). " LoadResistance factor design of axially loaded pile based on load test results", Jl. Geotech.Geoenv.Eng., ASCE, Vol.134, No.8, pp.1106-1117.

[7] Hashash, Y. M. A., Hook, J. J., Schmidt, B. and Yao, J. C. (2001). "Seismic design and analysis of underground structures", Tunnelling and Underground Space Technology, Vol. 16, pp. 247-293.

[8] Kulhawy, F. H. (1975). "Stresses and displacements around openings in homogeneous rock", International Journal of Rock Mechanics and Mining Science \& Geomechanics Abstracts, Vol. 12, pp. 43-57.

[9] Naik, A, V. (1981). "Interactive analysis of tunnels in layered medium using finite and infinite elements", Ph.D. Thesis, 1981, IIT Bombay, India.

[10] Myers, R.H. and Montgomery, D.C. (1995). "Response surface methodology process and product optimization using design experiments", John Wiley \& Sons, New York.

[11] Gui, M.W. and Chien, M.C. (2006). "Blast-resistant analysis for a tunnel passing beneath Taipei Shongsan airport - a parametric study", Geotechnical and Geological Engineering, Vol. 24, pp. 227-248.

[12] American Concrete Institute, (2005). "Building code requirements for structural concrete (ACI 318-05)", ACI Committee 318, Michigan.

[13] Mollon, G., Dias, D and Soubra, A.H. (2009). "Probabilistic analysis of circular tunnels in homogeneous soil using response surface methodology", Journal of Geotechnical and Geoenvironmental Engineering, ASCE, Vol 135(9), pp. 1314-1325.

[14] National Geophysical Data Centre., University of California, Seismograph station, Berkeley, CA and
Institute of Earth Sciences of the Academia Sinica, Taipei, Taiwan. 